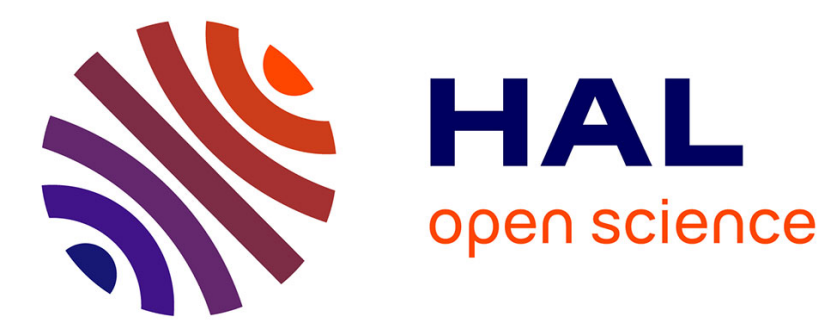

\title{
Polarized nonlinear nanoscopy of metal nanostructures
}

Naveen Kumar Balla, Carolina Rendon, Luong Mai Hoang, Pawel Karpinski, Esteban Bermudez Urena, Sophie Brasselet

\section{To cite this version:}

Naveen Kumar Balla, Carolina Rendon, Luong Mai Hoang, Pawel Karpinski, Esteban Bermudez Urena, et al.. Polarized nonlinear nanoscopy of metal nanostructures. ACS photonics, 2017, 7, pp.292-301. 10.1021/acsphotonics.6b00635 . hal-01537953

\section{HAL Id: hal-01537953 \\ https://hal.science/hal-01537953}

Submitted on 10 Apr 2020

HAL is a multi-disciplinary open access archive for the deposit and dissemination of scientific research documents, whether they are published or not. The documents may come from teaching and research institutions in France or abroad, or from public or private research centers.
L'archive ouverte pluridisciplinaire HAL, est destinée au dépôt et à la diffusion de documents scientifiques de niveau recherche, publiés ou non, émanant des établissements d'enseignement et de recherche français ou étrangers, des laboratoires publics ou privés. 


\title{
Polarized nonlinear nanoscopy of metal nanostructures
}

\author{
Naveen Kumar Balla 1,\&, Carolina Rendon-Barraza 1,\&, Luong Mai Hoang 1,2, Pawel Karpinski ${ }^{3}$, \\ Esteban Bermúdez-Ureña ${ }^{4}$ and Sophie Brasselet ${ }^{*}, 1$ \\ ${ }^{1}$ Institut Fresnel, CNRS, Aix-Marseille Université, Ecole Centrale Marseille, Domaine Universitaire St Jérôme, \\ 13013 Marseille, France \\ ${ }^{2}$ Current address: Department of Physics and Astronomy, the University of Georgia, Athens, GA 30602, USA \\ ${ }^{3}$ Current address: Department of Applied Physics, Chalmers University of Technology, S-412 96 Goteborg, \\ Sweden \\ ${ }^{4}$ ICFO-Institut de Ciencies Fotoniques, Barcelona Institute of Science and Technology, 08860 Castelldefels \\ (Barcelona), Spain
}

*Email: sophie.brasselet@fresnel.fr

\begin{abstract}
Nonlinear signals from metal nanostructures are known to be highly polarization-dependent, due to the intrinsic vectorial nature of nonlinear optical coupling. Nonlinear optical polarization responses contain important information on the near-field properties of nanostructures; however they remain complex to monitor and to model at the nano-scale. Polarization resolved nonlinear optical microscopy can potentially address this question, however the recorded signals are generally averaged over the diffraction-limited size of a few hundreds of nanometers, thus missing the spatial specificity of the nanostructure's optical response. Here we present a method of polarized nanoscopy that exploits subdiffraction resolution information down to a few tens of nanometer. Even though the resulting image is diffraction-limited, the information gained by polarization-induced modulation provides a higher level of selectivity that is directly related to vectorial optical responses at a scale below the diffraction limit. We show that polarized nonlinear nanoscopy permits to spatially map the vectorial nature of plasmonic nonlinear optical interactions in nanostructures.
\end{abstract}

\section{Introduction}

Metal nanostructures are essential building blocks for nanophotonics, with the unique capacity to tailor optical fields at the nanometric scale. One of the key factors that control their nanoscale optical properties is the polarization of incident electromagnetic fields, which influences the amplitude and polarization of scattered fields. By varying the excitation polarization, one can not only tune the spectral properties of metal nanostructures of complex shapes ${ }^{1,2}$, but also the spatial and vectorial properties of their local fields at the nanoscale. Controlling these properties has opened new routes for optimized biosensors, contrast agents and nano-antennas ${ }^{3-5}$, dedicated to new device functions ${ }^{6-11}$, but also for the exploration of fundamental light matter coupling properties ${ }^{12-14}$. The polarization sensitivity of metal nanostructures at the nanoscale is delicate to minitor, despite being well understood from numerical simulations ${ }^{15-17}$. Only a few experimental techniques give access to vectorial properties of the optical fields in the vicinity of metal nanostructures. Near field scanning optical microscopy ${ }^{18-24,30}$, cathodoluminescence microscopy and photoemission electron microscopy can image local fields with high spatial resolution ${ }^{25-29}$. However these techniques are delicate to implement, can be invasive and need special sample preparation which often does not fit the working conditions of optical devices. Polarized dark field polar pattern recognition ${ }^{30}$ has been implemented as a less invasive method and is able to report on nanoparticles' symmetry, but is not compatible with pure imaging. 
Nonlinear optical interactions can bring a way to report local fields' properties. Not only nonlinear quadratic effects are intrinsically background-free, their high order dependence on the incident field also makes them fine probes for polarization effects as compared to linear optics ${ }^{17,31-36}$. Second Harmonic Generation (SHG) and two photon luminescence (TPL) have been reported to be particularly sensitive to vectorial coupling between the excitation fields and the nanostructures ${ }^{34,37,38}$. However such methods are intrinsically limited in optical resolution to a few hundreds of nanometers (typically 200 to $300 \mathrm{~nm}$ ). This spatial averaging complexifies the retrieval of vectorial, local information $31,34,35$.

In this work, we show the possibility to access nanoscale vectorial information in metal nanostructures using far field two-photon excitation microscopy. This method combines the high spatial sampling capabilities of laser scanning microscopy and the polarization sensitivity of nonlinear responses to subresolution features and plasmon modes in the nanostructures. Accessing such polarized information allows filtering-out local symmetry responses that are directly related to the vectorial nature of the nonlinear radiation sources in the nanostructures. We show that the obtained sub-diffraction scale mapping relates to the vectorial nanoscale distribution of plasmon modes in the structure.

\section{Results and discussion}

Gold nanoparticles (NPs) of star shapes with four arms were fabricated by Electron Beam Lithography (EBL) (Figure 1a inset) (see Methods section). Their size is $200 \mathrm{~nm}$, which is below the transverse diffraction limit of our microscope $(250 \mathrm{~nm})$. Different shapes of nanoparticles were designed to tune the spatial distribution as well as vectorial properties of confined optical fields; aspect ratios of horizontal to vertical arm sizes vary from 1:1 (NP1), 1:0.93 (NP2), 1:0.86 (NP3), 1:0.78 (NP4), to 1:0.71 (NP5) (see Figure S1 in the Supplementary Information (SI)). The plasmon resonances of those particles range between $800 \mathrm{~nm}$ and $1100 \mathrm{~nm}$ (See Methods section and Figure S1 in the SI). The particles were positioned on the glass coverslip in a repetitive pattern, which allows not only measuring a large number of them, but also to precisely know their orientation, which is an important parameter in vectorial investigations.

Polarization resolved two-photon microscopy has been described in detail elsewhere (see Methods section) ${ }^{32,34,39}$. Briefly, a $150 \mathrm{fs}$ excitation source with wavelength in the range $800-1100 \mathrm{~nm}$ is used in a epi-geometry two-photon scanning microscope (Figure 1a) and focused by a high numerical aperture objective that provides a lateral optical resolution of $250 \mathrm{~nm}$. The nonlinear signal collected by the objective is recorded by two photomultiplier tubes detecting separately second harmonic generation (SHG) and two-photon luminescence (TPL) signals. The incident linear polarization angle $\alpha$ (relative to the horizontal sample plane axis X, as defined in Figure 1a) is rotated by an achromatic half-wave plate mounted on a motorized rotational mount, in steps of $10^{\circ}$ over the range of $0^{\circ}-170^{\circ}$. At each incident polarization angle, an SHG/TPL image of size $4 \mu \mathrm{m} \times 4 \mu \mathrm{m}$ is formed, covering a field of view of four particles. A pixel size of $40 \mathrm{~nm}$ is chosen to intentionally over-sample the images in view of the resolution gained by the polarization modulation obtained. In the obtained polarimetric image stack, each pixel contains a signal modulation that thus reports the polarization dependent signal (P-SHG/TPL) from the particle, at this pixel position.

The principle of P-SHG/TPL nanoscopy is to analyze the polarization-dependent SHG or TPL intensity $\mathrm{I}(\alpha)$ per pixel of the scanned image. The rotation of the incident polarization varies the spatial repartition of confined fields in the nanostructure and hence of nonlinear images (Figures $1 \mathrm{~b}-\mathrm{d}$ ) ${ }^{34}$, even though the total intensity (sum over all incident polarization angles) stays as a symmetric diffraction limited spot (Figure 1e). As the particle is scanned by the focused incident optical beam, each pixel signal results from a nonlinear coupling at the overlap between the nanoparticle and the diffraction-limited spot at this particular pixel position. It thus reveals information of the sub-diffraction scale orientational organization of nonlinear induced dipoles within this focal spot. In order to access to such sub-diffraction 
pixel size accuracy, images are corrected for lateral spatial drift, which arises due to mechanical drift and slight spot position motion during the course of the half wave plate rotation that ensures polarization tuning (Figure 1f) (see Methods).

a)

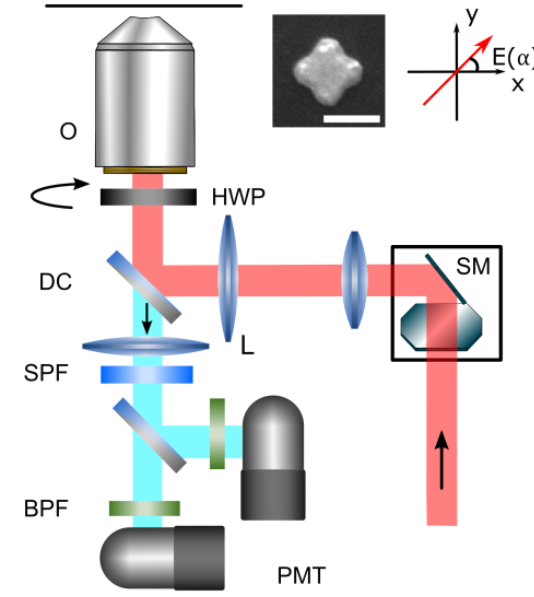

b)

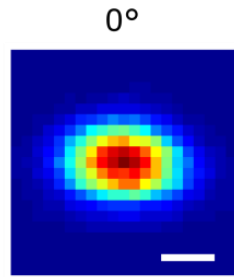

e)

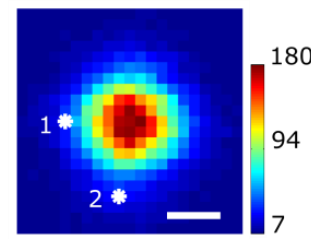

c)

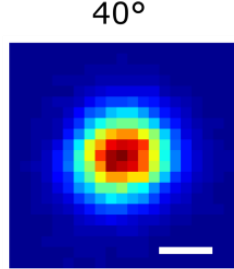

f)

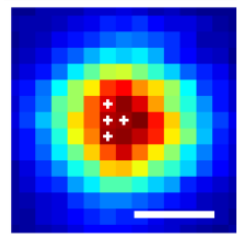

d)

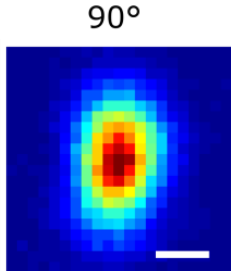

g)

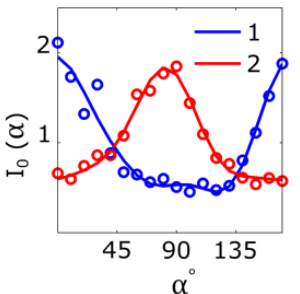

Figure 1. a) Experimental set-up. DC: Dichroic Mirror, SM: scan mirrors, L: lens, HWP: half wave plate, SPF: short pass filter, BPF: band pass filter, PMT: photo-multiplier tube. Inset: scanning Electron Microscopy (SEM) images of the nanoparticles of symmetric four arm shape (NP1). b-d) SHG images, at $800 \mathrm{~nm}$ excitation wavelength, of a NP1 particle for different incident polarizations directions with respect to the horizontal axis in the sample frame: b) $0^{\circ}$, c) $40^{\circ}$ and d) $90^{\circ}$. e) Total SHG intensity summed over all incident polarizations. f) Zoom on the total SHG intensity image showing the spatial drift of center during measurement, as pointed by white crosses representing the image center position for four different incident polarizations. g) Polarization dependence of the SHG signal at locations 1 and 2 shown in e). Scale bar (a-f): 200nm. Pixel size (b-f): 40nm.

Irrespective of the nature of the coupling process that yields SHG or TPL photons, the resulting $\mathrm{I}(\alpha)$ polarization dependent response (Figure 1g) is a fourth power dependence of the incident field $E(\alpha)$, which can be written as a decomposition onto circular functions of second and fourth orders ${ }^{32,39}$ :

$$
\mathrm{I}(\alpha) / \mathrm{I}_{0} \propto 1+a_{2} \cos 2 \alpha+b_{2} \sin 2 \alpha+a_{4} \cos 4 \alpha+b_{4} \sin 4 \alpha
$$

Here the incident field is assumed to be uniform in the focal spot with coordinates in the sample frame $(\mathrm{X}, \mathrm{Y}, \mathrm{Z}): E(\alpha)=(\cos \alpha, \sin \alpha, 0)$. This supposes that the field does not exhibit any longitudinal contribution along $\mathrm{Z}$, which is a strong approximation especially at high numerical aperture focusing ${ }^{40}$. Previous studies have shown that such contribution is generally negligible for dielectric particles lying in the sample plane ${ }^{41}$, however here the longitudinal coupling from the metal planar surface of the nanoparticle might add an isotropic contribution to $\mathrm{I}(\alpha)$ which needs to be accounted for in the data interpretation. The $\left(a_{2}, b_{2}, a_{4}, b_{4}\right)$ coefficients are retrieved experimentally by projection on circular functions, following $a_{2}=2 \sum_{k} \mathrm{I}\left(\alpha_{k}\right) \cos 2 \alpha_{k} / \sum_{k} \mathrm{I}\left(\alpha_{k}\right)$ and similar relations for other coefficients ${ }^{39,42}$. $I_{0}=\sum_{k} \mathrm{I}\left(\alpha_{k}\right)$ denotes the total intensity, summed over all incident polarization angles $\alpha_{k}$. This decomposition encompasses all possible processes that are responsible for the polarization-dependent modulation of the SHG or TPL signals, be it of dipolar or quadrupolar nature ${ }^{15}$. The deduced second and 
fourth order coefficients are direct signatures of the symmetry of the vectorial coupling that locally takes place in the particle, occurring from local dipolar origin of multiple orientations, or from responses of higher orders of symmetry, including retardation effects. These orders can be assembled into amplitudes $\left(I_{2}, I_{4}\right)$ and orientations $\left(\varphi_{2}, \varphi_{4}\right)^{39,42}$ :

$$
\begin{aligned}
& I_{2}=\sqrt{a_{2}^{2}+b_{2}^{2}}, \quad I_{4}=\varepsilon_{4} \sqrt{a_{4}^{2}+b_{4}^{2}} \\
& \varphi_{2}=\frac{1}{2} \arctan \frac{b_{2}}{a_{2}}, \quad \varphi_{4}=\frac{1}{4} \arctan \frac{b_{4}}{a_{4}}
\end{aligned}
$$

With $\varphi_{4}$ given modulo $\pi / 2 . \quad \varepsilon_{4}=\cos \left(4\left(\varphi_{4}-\varphi_{2}\right)\right)$ sets the sign of $I_{4}$ such that the fourth order response lies either along the second order $\left(I_{4}>0\right)$ response or $\pi / 4$ phase shifted $\left(I_{4}<0\right)^{43}$. In the simplest system of a pure 1D dipolar response along a direction $\theta$ with respect to $\mathrm{X}$ (for instance from a metal nanorod oriented in the plane excited for its longitudinal plasmon mode, in the dipolar approximation), the onedimensional susceptibility tensor that contributes to the nonlinear response leads to I $(\alpha) \propto \cos ^{4}(\theta-\alpha)$, which yields $\left(I_{2}=1.35, I_{4}=0.38, \varphi_{2}=\varphi_{4}=\theta\right)$ (see Figure S2 in SI). These numbers serve, in what follows, as reference values for nanoparticles studies. If multiple dipole directions or multipolar nonlinear responses are present within the focal volume, the measured symmetry orders are expected to depart from these 1D reference values (see Figure S2 in SI). Altogether, $\left(I_{2}, I_{4}\right)$ and $\left(\varphi_{2}, \varphi_{4}\right)$ are direct signatures, as reported from nonlinear scattering, of local plasmonic properties of nanostructures. $I_{2}$ is in particular characteristic of local anisotropy, while $I_{4}$ represents the degree of multipolar symmetry in the involved nonlinear coupling.

The P-SHG/TPL nanoscopy analysis consists of performing this symmetry decomposition, not for an averaged nanoparticle signal, but at each pixel location formed by point scanning microscopy. The data are rendered as oversampled images, using a pixel size of $40 \mathrm{~nm}$ pixel. Data are systematically averaged over 32 images, in order to gain an estimation quality for the retrieved parameters. The signal level is set with a lower limit that ensures standard deviations lower than 0.01 on the $\left(I_{2}, I_{4}\right)$ parameters, with possible systematic bias that is pre-calibrated (see Figure S3 in the SI) ${ }^{42}$.

Figure 2 shows the result of a P-SHG analysis at $800 \mathrm{~nm}$ excitation on a symmetric four arm particle (NP1). The total intensity image $\left(I_{0}\right)$ resembles a symmetric spot without any specific feature, as expected from a nanoparticle of size lower than the diffraction limit (Figure 2a). At each pixel position, symmetry components $\left(I_{2}, I_{4}\right)$ and their orientations $\left(\varphi_{2}, \varphi_{4}\right)$ are depicted as a stick encoded with $\left(I_{2}, I_{4}\right)$ as a color and $\left(\varphi_{2}, \varphi_{4}\right)$ as an orientation relative to the horizontal sample axis (Figure $2 \mathrm{~b}, \mathrm{c}$ ).

a)

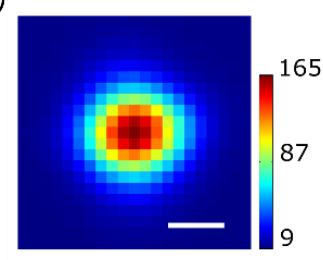

f)

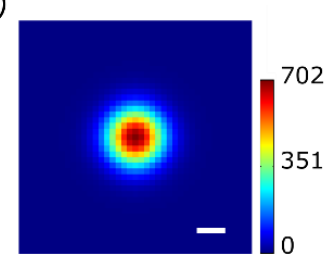

b)

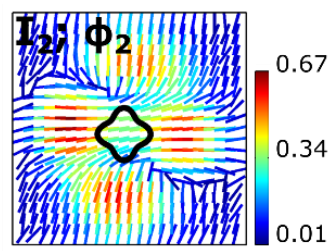

g)

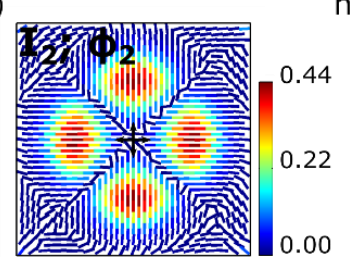

c)

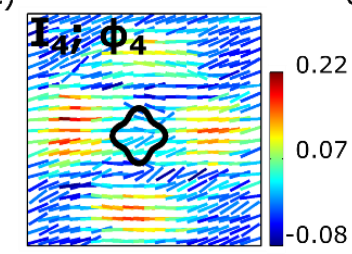

h)

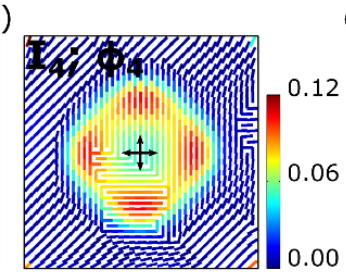

d)

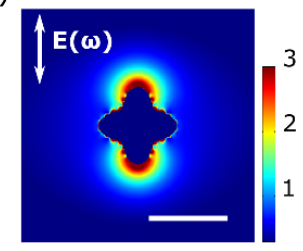

e)

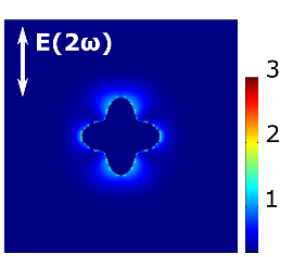


Figure 2. P-SHG response in a $200 \mathrm{~nm}$ size four arm symmetric structure (NP1) at $800 \mathrm{~nm}$ excitation. a) Total intensity image ( $I_{0}$ coefficient of the intensity circular decomposition). b) Second order $\left(I_{2}, \varphi_{2}\right.$ coefficients) image, representing for each pixel a stick with orientation $\varphi_{2}$ relative to the horizontal axis and color coded with $I_{2}$. Contour plots of the nanoparticles shape are superimposed with the images at real scale. Pixel size: $40 \mathrm{~nm}$. c) Similar representation for $\left(I_{4}, \varphi_{4}\right)$ (the angle $\varphi_{4}$ is determined modulo $\pi / 2$ ). d) Simulated map (using Dipole Discrete Approximation, see Methods) of the near field scattering of the incident optical field amplitude at $800 \mathrm{~nm}$. e) Same map at the SHG wavelength $400 \mathrm{~nm}$. f) Simulated total intensity image for a structure made of a planar center and four dipoles along the four arms of the nanoparticle (see Methods). The distance between the dipoles is $200 \mathrm{~nm}$, the physical size of the dipoles is $40 \mathrm{~nm}$, and the center of the structure, of size $40 \mathrm{~nm}$, is considered as exhibiting a SHG isotropic response, as expected from a pure longitudinal response. g) Resulting $\left(I_{2}, \varphi_{2}\right)$ map. h) Resulting $\left(I_{4}, \varphi_{4}\right)$ map. Scale bars : $200 \mathrm{~nm}$. Pixel size : $40 \mathrm{~nm}$.

Figures $2 \mathrm{~b} \& \mathrm{c}$ clearly reveal features that are not present in the intensity image, with shapes that directly correlate with the four arms structure of the nanoparticle. The $\left(I_{2}, I_{4}\right)$ values at the center of the image are low, which is expected since when the excitation spot is positioned at the center of the particle, it induces nonlinear dipoles distributed centrosymmetrically, coming from surface dipoles that are orthogonal to the sample plane, or to symmetrically arranged surface dipoles. In contrast, when the excitation spot is centered away from the particle edge but still excites its contour, it interacts mostly with distributions of dipoles aligned in a well-defined direction, here along the particle arms were the optical fields are expected to be strong and anisotropic. Numerical simulations (performed with Dipole Discrete Approximation, see Methods) $)^{44,45}$ confirm that at the incident optical wavelength $800 \mathrm{~nm}$ for such nanoparticle, the scattered field is confined and strong at the tip of the arms along the field's polarization (Figure 2d). Note that at the SHG generated wavelength $400 \mathrm{~nm}$, there is no specific feature in the scattering of the harmonic generated light (Figure 2e), therefore the observed anisotropic features are likely to come from a one photon resonance process. Remarkably, the spatial extent of the $\left(I_{2}, \varphi_{2}\right)$ and $\left(I_{4}, \varphi_{4}\right)$ images is bigger than the diffraction limit size. This is the result of the fact that polarization dependent modulation survives to a much larger spatial extent than the pure intensity image, since even low signals have the ability to exhibit strong intensity modulation. This is also illustrated in Figure $1 \mathrm{~g}$ where polarization modulated signals are clearly visible for pixels that lie at the edge of the nanoparticle's diffraction limit image.

The presence of distinct, highly dipolar responses at the tip of the arms shows that the structure is expanded enough to confine optical fields away from the particle center, suggesting a negligible coupling between arms. However the values of $\left(I_{2}, I_{4}\right)$ at the tip positions of the structure reach magnitudes that are slightly lower than those of single, isolated 1D dipolar symmetry mentioned above $\left(I_{2}=1.35, I_{4}=\right.$ 0.38). Different factors can explain this deviation: induced dipoles at the tips of the structure might not be organized in a pure 1D symmetry assembly (see Figure S2 in SI), the longitudinal contribution from the metal surface might be non-negligibe, and phase shifts between the radiation of coherent dipoles from opposite tips might exist. This last effect can modify the local symmetry of the polarization response by introducing relative phases correlated with induced dipoles positions, which plays a role in the nonlinear radiation from the nanoparticle ${ }^{46}$.

More quantitative information can be gained from a simple phenomenological model. We suppose four 1D dipolar structures of $40 \mathrm{~nm}$ size placed at the tip positions of the nanoparticle, and pointing along the tips of the arms of the structure. A central structure is added with polarization-independent response, mimicking a longitudinal dipole contribution from the metal surface of the nanoparticle. The P-SHG signal is modeled as a coherent superposition of those nonlinear dipolar responses, convolved by the point spread function (PSF) of the excitation spot (see Methods). This simple model reproduces the essential elements of the experimental results (Figure 2e-g): the SHG total intensity image is diffraction limited, 
and four spots are visible in the $\left(I_{2}, \varphi_{2}\right)$ and $\left(I_{4}, \varphi_{4}\right)$ images, with $\varphi_{2}$ aligned along the dipoles directions. We noticed that the magnitude of $I_{2}$ in the anisotropic spots increases with the distance between the dipoles; a distance of about $200 \mathrm{~nm}$ leads to $I_{2}$ values close to the experimental ones. Even though this model does not reproduce the full characteristics of the electromagnetic modes in the particle, it shows that P-SHG is able to report the dimension and local symmetry of the structure. From those results, it is clear that a sole measurement of the P-SHG response averaged at the particle center would miss the spatial specificity of this response and possibly bias its interpretation. Interestingly, experimental P-TPL images exhibit very similar properties (see Figure S4 in SI), which supports the fact that both SHG and TPL processes are governed by common radiation sources. Note that the incoherent nature of the emission is not probed here since the detection is unpolarized ${ }^{34}$.

To investigate how sensitive is P-SHG to slight variations of the nanoparticles shape, we introduced deviations from the symmetric four arms structure, and studied their P-SHG wavelength dependence. On decreasing progressively the size of the arms in one direction (Figure 3), the $\left(I_{2}, \varphi_{2}\right)$ maps appear quickly dominated by a preferential direction along the shorter particle arms, suggesting a transverse plasmon mode in the structure. The $I_{2}$ magnitudes reached for NP2 and NP3 particles are higher than for the four arm NP1 structure, due to stronger plasmon coupling between tips of the shorter arms. $I_{2}$ then decreases for the nano-rod like NP5 structure, due to the loss of anisotropy. $I_{4}$ follows similar trends. Because of the stronger transverse plasmon coupling as compared to NP1 where arms are isolated, both $\left(I_{2}, \varphi_{2}\right)$ and $\left(I_{4}, \varphi_{4}\right)$ maps lose their contrasted picture. Overall when the transverse arms diminish in length, the excitation fields lose their localized nature; this feature was not observable from pure intensity images, which look identical whatever the type of particle is imaged.

a)

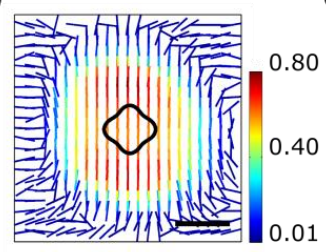

b)

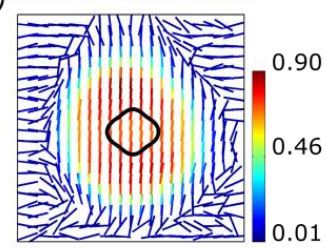

c)

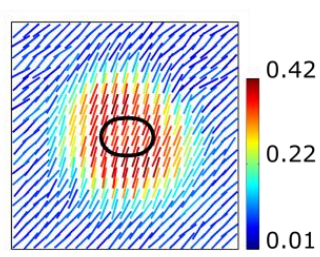

d)

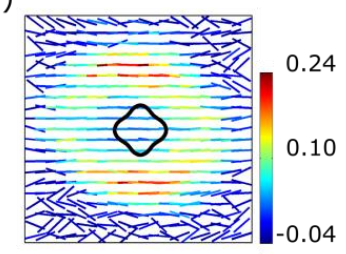

e)

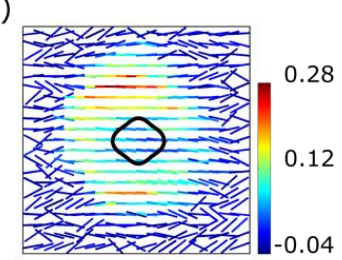

f)

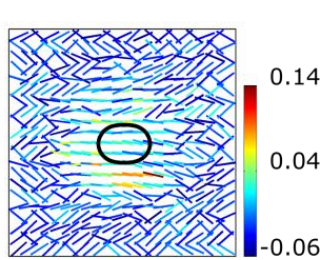

Figure 3. P-SHG results for nanoparticles made of four arms of various lengths. Left: second order $\left(I_{2}, \varphi_{2}\right)$ image, representing for each pixel a stick with orientation $\varphi_{2}$ relative to the horizontal axis and color coded with $I_{2}$. Right: similar representation for $\left(I_{4}, \varphi_{4}\right)$. Ratios of horizontal to vertical arm lengths are 1:0.93 (NP2) (a,d), 1:0.86 (NP3) (b,e) and 1:0.71 (NP5) (c,f). Contour plots of the nanoparticles shape are superimposed with the images at real scale. Scale bar: $200 \mathrm{~nm}$. 
Figure 3 shows that at $800 \mathrm{~nm}, \mathrm{P}-\mathrm{SHG}$ reflects a transverse plasmon mode. On moving the excitation wavelength from $800 \mathrm{~nm}$ to $1000 \mathrm{~nm}$, the linear excitation is expected to approach a longitudinal plasmon resonance (Figure 4). Upon incident wavelength change, the SHG efficiency $\left(I_{0}\right)$ of the studied particles is correlated to the linear excitation resonance both in wavelength dependence (Figure 4a) and in particles shape (Figure 4b), as expected from the effects of SHG dependence on the linear incident fields enhancements.
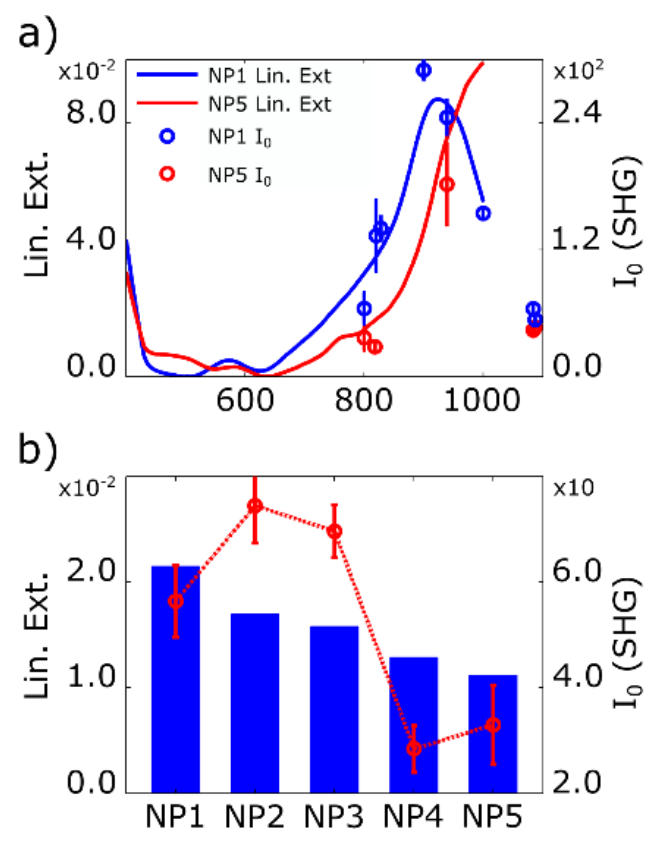

Figure 4. Effect of the incident wavelength on SHG efficiencies (four arms particles of different aspect ratios). a) Linear extinction of the NP1 and NP5 particles, together with their wavelength-dependent SHG efficiency $I_{0}$. b) Particle-shape dependence of the linear extinction (bars) and nonlinear efficiency (markers) close to $800 \mathrm{~nm}$. Characteristics of the aspect ratio of horizontal to vertical arms: 1:1 (NP1), 1:0.93 (NP2), 1:0.86 (NP3), 1:0.78 (NP4), 1:0.71 (NP4). Errors bars are standard deviation over 3 to 5 measurements, each of them including 32 measured images.

Tuning the excitation wavelength from $800 \mathrm{~nm}$ to $1000 \mathrm{~nm}$ also induces drastic modifications of the P-SHG response, both in symmetry orders magnitudes and orientations. Figure 5a shows that all types of particles do not follow the same trend. For the symmetric four-arm shape structure (NP1), while a 800 $\mathrm{nm}$ excitation shows well defined individual and separated hot spots as mentioned above (Figure $5 \mathrm{~b}$ ), increasing the excitation wavelength leads to a loss of contrast in the $I_{2}$ image (Figures $5 \mathrm{c} \& \mathrm{~d}$ ). This decrease is likely due to an increased coupling between the arms, similarly to what was observed in NP2 and NP3 particles (Figure 3). In the one-arm nanoparticles (NP5), the behavior follows an opposite trend, with an increase of $I_{2}$ with wavelength. At $800 \mathrm{~nm}$ excitation, the orientation $\varphi_{2}$ of second order dipoles is perpendicular to the particle, as seen above (Figure 5e). Changing the incident wavelength towards 1000 $\mathrm{nm}$ shifts this orientation to along the particle (Figures $5 \mathrm{f} \& \mathrm{~g}$ ), obviously shifting from the excitation of the transverse mode at $800 \mathrm{~nm}$ to the longitudinal mode at $1000 \mathrm{~nm}$. In this mode, the $\left(I_{2}, I_{4}\right)$ values obtained are close to those of a single isolated dipole, which evidences the strongly dipolar nature of the longitudinal plasmon mode. Intermediate shapes (NP2 to NP4) follow intermediate behaviors (see Figure S5 in SI). Note however that for all particles, a drop of $I_{2}$ and $I_{4}$ is noticed at the intermediate wavelength $820 \mathrm{~nm}$ (see Figures S6 and S7 in SI). This reveals the presence of a strong centrosymmetric contribution, which could originate from a quadrupolar mode $^{1}$ or from mixed dipolar excited modes which 
destructively interfere. Such behavior is usually delicate to observe in far field experiments, and P-SHG nanoscopy could be a way to reveal its complexity.

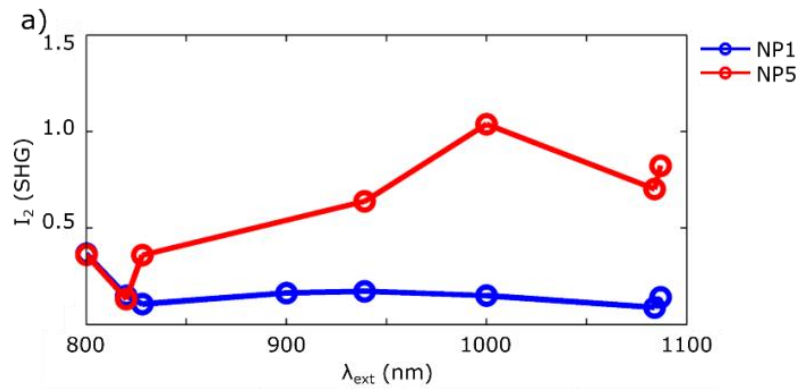

b)

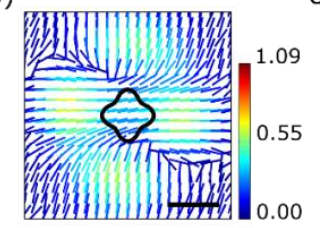

e)

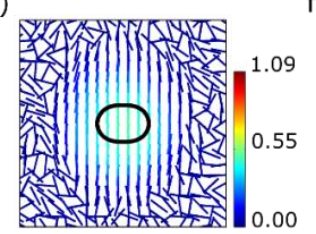

c)

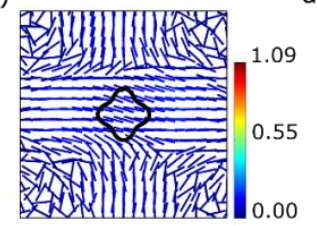

f)

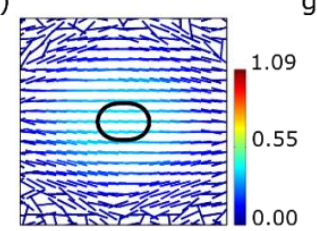

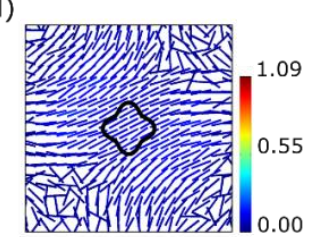

g)

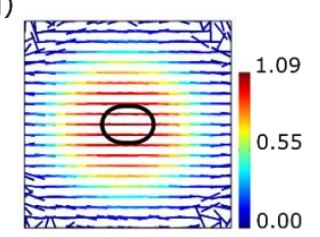

Figure 5. Effect of the incident wavelength on P-SHG from NP1 and NP5 particles. a) Maximum reachable $I_{2}$ as a function of wavelength of excitation for NP1 and NP5 particles. For the estimation of $I_{2}$, the mean of its 25 highest values over the P-SHG map is selected. b) $\left(I_{2}, \varphi_{2}\right)$ maps for NP1 and NP5 particles (shown with their contour), at $800 \mathrm{~nm}, 940 \mathrm{~nm}$ and $1000 \mathrm{~nm}$ excitation.

Smaller scales were finally analyzed by P-SHG nanoscopy. We used $45 \mathrm{~nm}$ length gold nanorods (aspect ratio 4.5), which longitudinal plasmon resonance lies at $850 \mathrm{~nm}$ (see Methods). At the incident wavelength $800 \mathrm{~nm}$, their SHG emission should thus behave as highly anisotropic with well defined $\left(I_{2}, I_{4}\right)$ values close to 1D dipoles as detailed above. Dropcasting a solution of nanorods on a microscope coverslip allowed collecting different types of behaviors from single nanorods to aggregated structures made of several nanorods, as illustrated in Figure 6.

a)

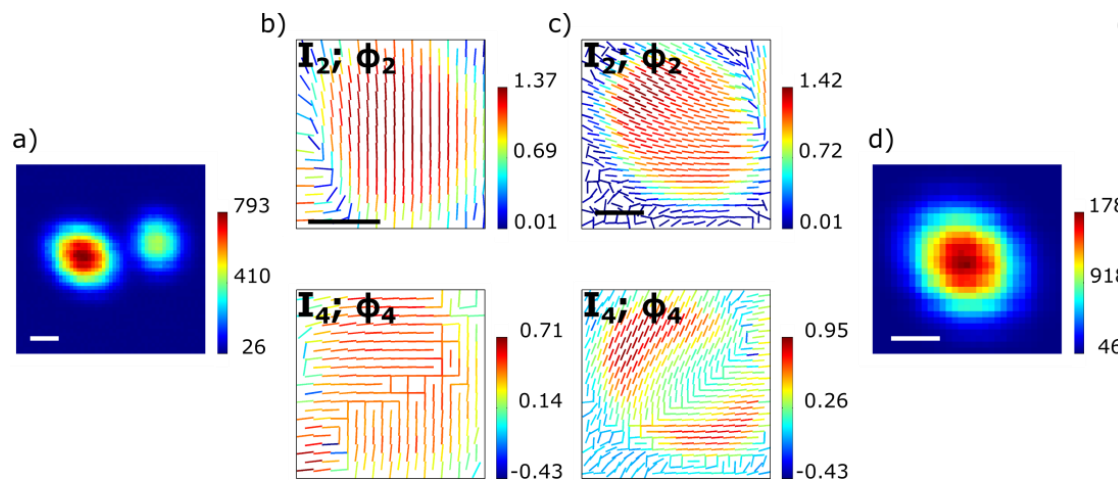

b) e)
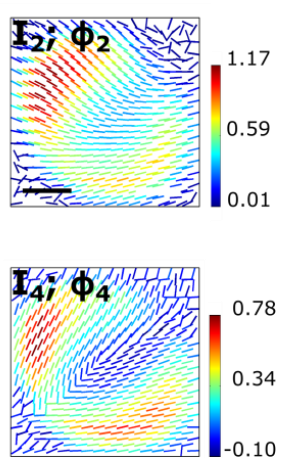
Figure 6. P-SHG nanoscopy in gold nanorods. a) Total SHG intensity $\left(I_{0}\right)$ on two structures made of dropcasted gold nanorods of size $45 \mathrm{~nm}$, under $800 \mathrm{~nm}$. b) $\left(I_{2}, \varphi_{2}\right)$ (top) and $\left(I_{4}, \varphi_{4}\right)$ (bottom) maps of the nanostructure visible on the right in the a) image. c) same maps for the nanostructure on the left in the a) image. d) Total SHG intensity $\left(I_{0}\right)$ of another nanostructure. e) same maps as b) and c) for this nanostructure. Scale bars: $200 \mathrm{~nm}$. Pixel size: $40 \mathrm{~nm}$.

In all the images represented here, the size of the SHG spot is close to the diffraction limit or slightly above it, and the SHG image does not permit to discriminate between one or several nanorod. P-SHG maps can reveal information that is not present in the intensity images, in particular highlighting the number of nanorods (at least a lower limit) and their respective orientations. In the specific examples of Figures $6 \mathrm{c} \& \mathrm{e}$, two orientations are clearly noticed from the $\left(I_{2}, \varphi_{2}\right)$ images. These nanorods are however in closer proximity for Figure 6c, since Figure 6e shows a $\left(I_{2}, \varphi_{2}\right)$ image that spreads on a larger size.

The $\left(I_{2}, \varphi_{2}\right)$ and $\left(I_{4}, \varphi_{4}\right)$ images of Figure 6 show that not only the relative orientations of nanorods can be potentially revealed by such a method, but also some insight can also be gained in their relative distance. In an image reminiscent of the four-arms nanoparticles described above, the $\left(I_{2}, \varphi_{2}\right)$ map would be indeed very homogeneous for closely interacting dipoles (e.g. case of the NP3 to NP5 particles), while distant dipoles reveal a drop of $I_{2}$ and $I_{4}$ at the center of the image (e.g. case of NP1). In order to confirm this trend, we simulated two nanorods at a distance of $40 \mathrm{~nm}$ to $120 \mathrm{~nm}$, with a relative orientation of $120^{\circ}$ in order to resemble the situations present in Figure 6. Using a similar approach as in Figure 2f-h, the nanorods are taken of $40 \mathrm{~nm}$ size, and of 1D pure dipolar symmetry, to mimic their longitudinal response. The resulting $\left(I_{2}, \varphi_{2}\right)$ and $\left(I_{4}, \varphi_{4}\right)$ maps depicted in Figure 7 show clear resemblance with the measured maps. In order to reproduce the drop of the $I_{4}$ value at the center of the structure, a phase shift has to be introduced in the emission of the two dipoles (similarly to taking a relative orientation of $-60^{\circ}$ without changing the relative spatial positions of the dipoles), evidencing the contribution from spatial retardance in the nonlinear coherent build up. This highlights, as above, the sensitivity of the method to phase.

a)

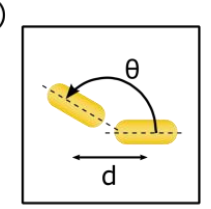

b)

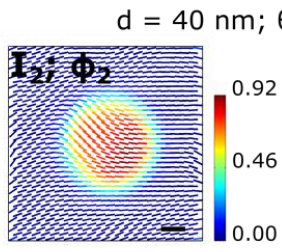

$\mathrm{d}=40 \mathrm{~nm} ; \theta=120^{\circ}$

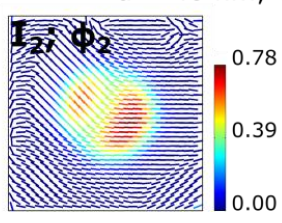

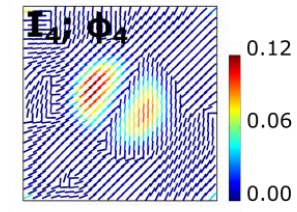

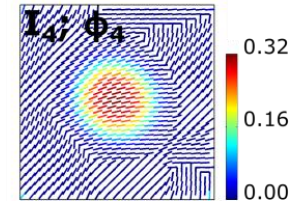

c) $\quad d=120 \mathrm{~nm} ; \theta=-60^{\circ}$
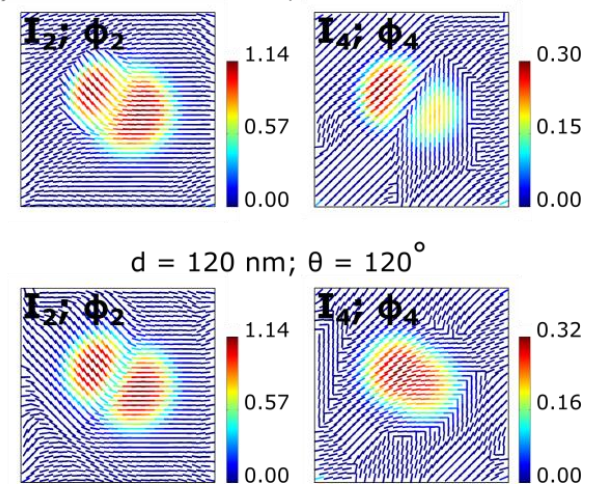

$\theta=120^{\circ}$

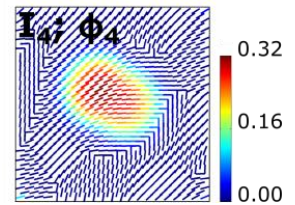

Figure 7. Simulated P-SHG nanoscopy in two gold nanorods of 1D symmetry. a) Schematics of the nanorods position and orientation: the distance between their center is $\mathrm{d}$, and the left nanorod is moved in the vertical direction by a distance $0.6 \mathrm{~d}$. Their relative orientation defined by $\theta$. b) $\left(I_{2}, \varphi_{2}\right)$ (left) and $\left(I_{4}, \varphi_{4}\right)$ (right) maps for the case $\mathrm{d}=40 \mathrm{~nm}, \theta=-60^{\circ}$ (or similarly $240^{\circ}$ ) (above) and $\theta=120^{\circ}$ (below). c) similar maps for the case $\mathrm{d}=120 \mathrm{~nm}, \theta=-60^{\circ}$ (above) and $\theta=120^{\circ}$ (below). Scale bar: $200 \mathrm{~nm}$. Pixel size: $40 \mathrm{~nm}$. 
The experimental data shown in Figure 6 are in best agreement with two dipoles distant by respectively about $40 \mathrm{~nm}$ (Figures $6 \mathrm{~b}$ and 7b, upper panel) and $120 \mathrm{~nm}$ (Figures 6e and 7c, upper panel). Using this theoretical model, we noticed that P-SHG nanoscopy is sensitive to nanometric scale distances between nanostructures, as long as relative orientations between nanorods stays higher than about $10^{\circ}$, similarly to what has been shown in polarization based super resolution microscopy ${ }^{47}$. At the lowest nanoparticles inter-distances, the highly confined fields generated by coupling could also be studied by P-SHG, with the advantage of accessing their vectorial properties. P-SHG nanoscopy can thus reveal considerable amount of information as compared to pure SHG imaging or to averaged P-SHG signals. Note that optical phase can play an important role in the observation of plasmon modes. Local phase shifts between dipolar modes can explain in particular the existence of nonlinear modes that do not necessarily reflect the linear picture ${ }^{16}$. Phase shifts between dipolar and higher order modes can also induce strong spectral modifications of nano-antennas such as the appearance of Fano resonances, as recently observed in oligomeric structures ${ }^{48-51}$. Observation of spatial phase profiles is however delicate and has so far relied on indirect directionality measurements ${ }^{52,53}$ or near field measurements ${ }^{21}$. This method is able to reveal such effect, and could be advantageously coupled to dark field spectroscopy and transmission electron microscopy to investigate the existence and vectorial nature of bonding and anti-bonding modes in multimeric nanorod structures $2,54-56$ or more complex oligomeric structures.

\section{Conclusion}

P-SHG/TPL nanoscopy exploits the spatial sensitivity of polarized responses of the nonlinear emission from metal nanostructures. Its sensitivity to local surface effects and plasmon mode symmetry has permitted to evidence the spatial and vectorial properties of local nonlinear radiation sources. Even though far field microscopy conditions are used, this method is sensitive to nanometric scales, allowing access to information that cannot be revealed by pure linear or nonlinear imaging. P-SHG nanoscopy could be extended to more complex structures such as coupled nano-antennas or arrays of particles, designed to engineer novel polarized optical properties. It can also be applied to any other optical contrasts, in particular nonlinear processes of higher orders ${ }^{57}$.

\section{Methods}

Nanoparticles fabrication by Electron Beam Lithography.

Glass substrates were capped with a conductive $10 \mathrm{~nm}$ ITO layer deposited by electron beam evaporation. For the lithography step, a 950 PMMA at 4\% (Microchem) solution was dissolved in Trichlorobenzene (3:1) and spincoated at $8000 \mathrm{rpm}$ for $60 \mathrm{sec}$, yielding a resist thickness of $\sim 120 \mathrm{~nm}$. The resist was baked at $175^{\circ} \mathrm{C}$ for $5 \mathrm{~min}$. The structures were defined by electron beam lithography in a FEI InspectF50 system at $30 \mathrm{keV}$ acceleration voltage. The geometry of the structures was defined by importing the corresponding formula describing the particle shape into the ELPHY plus design software from Raith (Raith Gmbh). After the EBL exposure, the sample was developed in a MIBK:IPA (1:3) mixture for 45 sec followed by immersion in IPA solution to stop the development. The substrate was finally dried with a $\mathrm{N}_{2}$ gun. A $40 \mathrm{~nm}$ Au film was thermally evaporated at a rate of $2 \AA / \mathrm{s}$. The lift-off was performed in acetone at $55^{\circ} \mathrm{C}$ during $1 \mathrm{~h}$ followed by rinsing in IPA before drying with $\mathrm{N}_{2}$.

\section{Linear extinction measurements}

The studied sample also comprised denser arrays of each of the structures to measure the extinction resonance of each design. The optical setup consists of a standard microscope in a bright field 
configuration. The illumination was performed from the bottom side of the sample by a $100 \mathrm{~W}$ halogen lamp with a linear polarizer aligned to the horizontal axis of the structures (see SEM images in SI Fig. S1) and a bright field condenser ( $0.1 \mathrm{NA}$ ). The transmitted light was collected with a bright field objective $(10 \times, 0.25 \mathrm{NA})$ and passed through a beam splitter into a CCD camera for alignment and to a spectrometer (Andor, Shamrock SR-303i) via an optical fiber (200 $\mu$ m diameter).

$P$-SHG/TPL experiment. The set-up is based on a two-photon scanning microscope which uses a Ti:Sapphire femtosecond laser and optical parametric oscillator (OPO) combination (150fs, $80 \mathrm{MHz})$, at excitation wavelength in the range 800-1500 $\mathrm{nm}$ (Coherent Inc.). The total power delivered to the sample lies in the range of $1.0-1.2 \mathrm{~mW}$. The laser beams sizes were expanded to fill the back aperture of the objective $(40 \mathrm{x} / 1.15 \mathrm{~W}$ Nikon Instruments Inc.). Imaging was performed on an inverted microscope (Eclipse Ti, Nikon Instruments Inc.) using a pair of galvonometric scan mirrors $6215 \mathrm{H}$, Cambridge Technology Inc.). The transverse optical resolution is estimated to be $250 \mathrm{~nm}$. The pixel size is typically $40 \mathrm{~nm}$ (field of view $4 \mu \mathrm{m} \times 4 \mu \mathrm{m}, 100 \times 100$ pixels). A polarized beam splitter (PBS252, Thorlabs Inc.) was used to make the excitation lasers linearly polarized after the scan mirrors. This ensured that the dichroic mirror (T770SPXR, AHF analysentechnik AG) received a $p$-polarized laser light during the entire experiment. After the dichroic mirror, the linear polarization angle of the excitation laser was controlled by an achromatic half-wave plate (AQWP10M-980, Thorlabs Inc.) mounted on a motorized rotational mount (PR50CC, Newport Corp.). A stack of images was acquired by changing the polarization angle of the lasers in steps of $10^{\circ}$ over the range of $0^{\circ}-170^{\circ}$. The nonlinear signal collected by the objective was filtered using a shortpass filter (ET750sp-2p8, Chroma Technology Corp.) before being detected by 2 Photomultiplier tubes (R9110, Hamamatsu Photonics K. K.) detecting separately SHG and TPL signal. Depending on the excitation wavelength, the SHG and TPL signals were separated using an appropriate dichroic beamsplitter. Each PMT detects signal over a specific spectral range depending on the combination of filters used: (SHG : 400/40, 442/46 or 500/24; TPL : bandpass BG37). The signal detection path consists of imaging pairs of lenses which image the back aperture of the objective on to the detector window. This configuration keeps the signal steady on the detector even though the excitation spot is continuously scanning the sample. Scanning and data acquisition was performed using an in-house LabVIEW (National Instruments Corp.) program ${ }^{58}$. The data is acquired by a data acquisition board (NI USB 6353, National Instruments Corp). Data analysis is performed using MATLAB (The MathWorks, Inc).

Data processing. In order to map $\left(I_{2}, I_{4}\right)$ and $\left(\varphi_{2}, \varphi_{4}\right)$ values in metal nanostructures with high accuracy, each nanoparticle was imaged 8 times and 4 nanoparticles of each type were measured, to give a data set of 32 images for each nanoparticle type. This data set includes particle to particle variation in fabrication process and image to image variation of the set up. For drift correction, the images are first denoised and the centroid of each nanoparticle image is calculated with pixel size accuracy. The obtained drift information is used to register all nanoparticle images in each polarization stack. Typical drifts during a P-SHG/TPL stack (duration 120 seconds) lie between $80 \mathrm{~nm}$ and $120 \mathrm{~nm}$. The pixels selected for data analysis exhibit intensity values that are at least two standard deviations above the mean background. For the data representation, all pixels within the represented ( $800 \mathrm{~nm} \times 800 \mathrm{~nm}$ ) window around the particles are plotted. Note that the total intensity level measured in all data is high enough to ensure high precision and negligible bias in the retrieved parameters (see Figure S3 in SI) ${ }^{42}$.

Nonlinear P-SHG models. Linear and nonlinear dipole moments induced by the external field are calculated using conjugate gradient algorithm. Near field and far field scattering distributions were calculated based on the dipoles positions placed on the particle theoretical shape contour. Given the large size of the measured nanoparticles, the dipole size was chosen to be $8 \mathrm{~nm}$. The particle was assumed to be surrounded by an ambient medium of refractive index 1.50. The permittivity of gold was obtained from a parametric relation ${ }^{59}$. The thickness of the structure was assumed to be $50 \mathrm{~nm}$. Excitation wavelength was set at $800 \mathrm{~nm}$ and vectorial excitation field distribution was calculated using Richards' integrals ${ }^{40}$. In the simulations, a focused laser spot (of diffraction limit size $250 \mathrm{~nm}$ ) was scanned over a square region of size $560 \mathrm{~nm}$, centered over the nanoparticle. The second harmonic signal was integrated over the 
objective aperture for each position of the focal spot. This process was repeated for different incident polarization angles to form a stack of simulated polarization resolved images. This data was treated in the same way as experimental data to yield symmetry order coefficients. Simulations where performed over a $13 \times 13$ pixel image and at each pixel, the incident polarization was changed from $0^{\circ}$ to $170^{\circ}$ in steps of $10^{\circ}$. The size of simulated pixels is $\sim 43 \mathrm{~nm}$ which matches closely with the experimental conditions. A total of 3042 simulations were required and any increase in size of simulated image would quadratically increase the computational expense.

Phenomenological P-SHG model. In the phenomenological approach, a collection of nonlinear dipoles is placed in the plane with individual size of $40 \mathrm{~nm}$. The problem is treated in 2D in the sample plane. The meshwork is sampled with a $40 \mathrm{~nm}$ pixel size. The optical response from individual nanoobjects is modeled based on a 1D dipolar response, or by an isotropic response to model the center of the particles. The final SHG response per pixel is computed by convolving the modeled SHG polarization dependence by a spatial Gaussian beam of $250 \mathrm{~nm}$ size. Noise is introduced by adding Poisson noise at a level typically met in experimental situations. The generated data are then treated similarly as for experimental data.

Gold nano-rods. Gold nanorods of length $45 \mathrm{~nm}$ and aspect ratio $=4.5$ (716839, Sigma Aldrich) were chosen for their longitudinal plasmon resonance at $850 \mathrm{~nm}$. Coverslips were thoroughly cleaned with ethanol and acetone before UV/Ozone treatment. To each coverslip, $5 \mu \mathrm{l}$ of 100 times diluted stock solution of gold nanorods was added and the coverslips were air dried before imaging.

\section{Supporting Information Available}

Figure S1, SEM images images and extinction spectra of the measured particles.

Figure $S 2,\left(I_{2}, I_{4}\right)$ values for a pure metal surface from 1D symmetry to multipolar symmetry.

Figure $\mathrm{S} 3$, estimation precision of the $\left(\mathrm{I}_{2}, \mathrm{I}_{4}\right)$ parameters, as a function of noise.

Figure S4, P-TPL data on the NP1 particle.

Figure $S 5,\left(I_{2}, \varphi_{2}\right)$ maps of NP1-5 particles as a function of excitation wavelength.

Figure S6, Wavelength dependence of $\mathrm{I}_{4}$ in NP1 \& NP5 particles, including $\left(\mathrm{I}_{4}, \varphi_{4}\right)$ maps.

Figure S7, $\left(\mathrm{I}_{4}, \varphi_{4}\right)$ maps of NP1-5 particles as a function of excitation wavelength.

\section{Author information}

Corresponding Author

*E-mail: sophie.brasselet@fresnel.fr

Author Contributions \& N. K. Balla and C. Rendon-Barraza contributed equally to the work

Notes The authors declare no competing financial interest.

\section{Acknowledgments}

The authors thank P. Ferrand for assistance in the instrumentation development as well as J. Duboisset and H. Rigneault for discussions. E.B.-U acknowledges support from Fundació Privada Cellex, the Spanish 
Ministry of Economy and Competitiveness, through the 'Severo Ochoa' Programme for Centres of Excellence in R\&D (SEV-2015-0522), the FPI fellowship from the Spanish MICIIN and Universidad de Costa Rica. This work was supported by the European Commission, Research Executive Agency Marie Curie Actions 607842 FINON ITN-2013.

\section{References}

(1) Verellen, N.; Van Dorpe, P.; Vercruysse, D.; Vandenbosch, G. A. E.; Moshchalkov, V. V. Dark and Bright Localized Surface Plasmons in Nanocrosses. Opt. Express 2011, 19 (12), 11034-11051.

(2) Dopf, K.; Moosmann, C.; Kettlitz, S. W.; Schwab, P. M.; Ilin, K.; Siegel, M.; Lemmer, U.; Eisler, H.-J. Coupled T-Shaped Optical Antennas with Two Resonances Localized in a Common Nanogap. ACS Photonics 2015, 2 (11), 1644-1651.

(3) Ming, T.; Zhao, L.; Yang, Z.; Chen, H.; Sun, L.; Wang, J.; Yan, C. Strong Polarization Dependence of Plasmon-Enhanced Fluorescence on Single Gold Nanorods. Nano Lett. 2009, 9 (11), 3896-3903.

(4) Heeg, S.; Fernandez-Garcia, R.; Oikonomou, A.; Schedin, F.; Narula, R.; Maier, S. A.; Vijayaraghavan, A.; Reich, S. Polarized Plasmonic Enhancement by Au Nanostructures Probed through Raman Scattering of Suspended Graphene. Nano Lett. 2013, 13 (1), 301-308.

(5) Ghenuche, P.; Mivelle, M.; De Torres, J.; Moparthi, S. B.; Rigneault, H.; Van Hulst, N. F.; GarcíaParajó, M. F.; Wenger, J. Matching Nanoantenna Field Confinement to FRET Distances Enhances Förster Energy Transfer Rates. Nano Lett. 2015, 15 (9), 6193-6201.

(6) Jourlin, Y.; Tonchev, S.; Tishchenko, a V; Pedri, C.; Veillas, C.; Parriaux, O.; Last, a; Lacroute, Y. Spatially and Polarization Resolved Plasmon Mediated Transmission through Continuous Metal Films. Opt. Express 2009, 17 (14), 12155-12166.

(7) Yang, J.; Zhang, J. Subwavelength Quarter-Waveplate Composed of L-Shaped Metal Nanoparticles. Plasmonics 2011, 6 (2), 251-254.

(8) Lerman, G. M.; Levy, U. Pin Cushion Plasmonic Device for Polarization Beam Splitting, Focusing, and Beam Position Estimation. Nano Lett. 2013, 13 (3), 1100-1105.

(9) Shafiei, F.; Wu, C.; Wu, Y.; Khanikaev, A. B.; Putzke, P.; Singh, A.; Li, X.; Shvets, G. Plasmonic NanoProtractor Based on Polarization Spectro-Tomography. Nat. Photonics 2013, 7 (5), 367-372.

(10) Trevino, J.; Walsh, G. F.; Pecora, E. F.; Boriskina, S. V; Dal Negro, L. Photonic-Plasmonic-Coupled Nanoantennas for Polarization-Controlled Multispectral Nanofocusing. Opt. Lett. 2013, 38 (22), 4861-4863.

(11) Andersen, S. K. H.; Pors, A.; Bozhevolnyi, S. I. Gold Photoluminescence Wavelength and Polarization Engineering. ACS Photonics 2015, 2 (3), 432-438.

(12) Aeschlimann, M.; Bauer, M.; Bayer, D.; Brixner, T.; De Abajo, F. J. G.; Pfeiffer, W.; Rohmer, M.; Spindler, C.; Steeb, F. Adaptive Sub-Wavelength Control of Nanoscopic Fields. In Conference on Quantum Electronics and Laser Science (QELS) - Technical Digest Series; 2007.

(13) Biagioni, P.; Savoini, M.; Huang, J. S.; Duò, L.; Finazzi, M.; Hecht, B. Near-Field Polarization Shaping by a near-Resonant Plasmonic Cross Antenna. Phys Rev B 2009, 80, 153409.

(14) Valev, V. K.; Silhanek, A. V; Verellen, N.; Gillijns, W.; Van Dorpe, P.; Aktsipetrov, O. A.; Vandenbosch, G. A. E.; Moshchalkov, V. V; Verbiest, T. Asymmetric Optical Second-Harmonic Generation from Chiral \$G\$-Shaped Gold Nanostructures. Phys. Rev. Lett. 2010, 104 (12), 127401.

(15) Bachelier, G.; Butet, J.; Russier-Antoine, I.; Jonin, C.; Benichou, E.; Brevet, P. F. Origin of Optical Second-Harmonic Generation in Spherical Gold Nanoparticles: Local Surface and Nonlocal Bulk Contributions. Phys. Rev. B - Condens. Matter Mater. Phys. 2010, 82 (23).

(16) Berthelot, J.; Bachelier, G.; Song, M.; Rai, P.; Colas des Francs, G.; Dereux, A.; Bouhelier, A. Silencing and Enhancement of Second-Harmonic Generation in Optical Gap Antennas. Opt. Express 2012, 20 (10), 10498.

(17) Butet, J.; Brevet, P.-F.; Martin, O. J. F. Optical Second Harmonic Generation in Plasmonic Nanostructures: From Fundamental Principles to Advanced Applications. ACS Nano 2015, 9 (11), 
$10545-10562$.

(18) Rang, M.; Jones, A. C.; Zhou, F.; Li, Z.-Y.; Wiley, B. J.; Xia, Y.; Raschke, M. B. Optical near-Field Mapping of Plasmonic Nanoprisms. Nano Lett. 2008, 8 (10), 3357-3363.

(19) Esteban, R.; Vogelgesang, R.; Dorfmüller, J.; Dmitriev, A.; Rockstuhl, C.; Etrich, C.; Kern, K. Direct near-Field Optical Imaging of Higher Order Plasmonic Resonances. Nano Lett. 2008, 8 (10), 31553159.

(20) Jones, A. C.; Olmon, R. L.; Skrabalak, S. E.; Wiley, B. J.; Xia, Y. N.; Raschke, M. B. Mid-IR Plasmonics: Near-Field Imaging of Coherent Plasmon Modes of Silver Nanowires. Nano Lett. 2009, 9 (7), 25532558.

(21) Alonso-Gonzalez, P.; Schnell, M.; Sarriugarte, P.; Sobhani, H.; Wu, C.; Arju, N.; Khanikaev, A.; Golmar, F.; Albella, P.; Arzubiaga, L.; Casanova, F.; Hueso, L. E.; Nordlander, P.; Shvets, G.; Hillenbrand, R. Real-Space Mapping of Fano Interference in Plasmonic Metamolecules. Nano Lett. 2011, 11 (9), 3922-3926.

(22) Dorfmüller, J.; Dregely, D.; Esslinger, M.; Khunsin, W.; Vogelgesang, R.; Kern, K.; Giessen, H. NearField Dynamics of Optical Yagi-Uda Nanoantennas. Nano Lett. 2011, 11 (7), 2819-2824.

(23) Habteyes, T. G.; Staude, I.; Chong, K. E.; Dominguez, J.; Decker, M.; Miroshnichenko, A.; Kivshar, Y.; Brener, I. Near-Field Mapping of Optical Modes on All-Dielectric Silicon Nanodisks. ACS Photonics 2014, 1 (9), 794-798.

(24) Singh, A.; Calbris, G.; Van Hulst, N. F. Vectorial Nanoscale Mapping of Optical Antenna Fields by Single Molecule Dipoles. Nano Lett. 2014, 14 (8), 4715-4723.

(25) Vesseur, E. J. R.; de Waele, R.; Kuttge, M.; Polman, A. Direct Observation of Plasmonic Modes in Au Nanowires Using High-Resolution Cathodoluminescence Spectroscopy. Nano Lett. 2007, 7 (9), 2843-2846.

(26) Kociak, M.; Nelayah, J.; Stephan, O.; Mazzucco, S.; Garcia de Abajo, F. J.; Bernard, R.; Colliex, C. Mapping Surface Plasmons on a Single Metallic Nanoparticle. In Frontiers in Optics 2008/Laser Science XXIV/Plasmonics and Metamaterials/Optical Fabrication and Testing; OSA Technical Digest (CD); Optical Society of America: Rochester, New York , 2008; p MMC1.

(27) Hrelescu, C.; Sau, T. K.; Rogach, A. L.; Jäckel, F.; Laurent, G.; Douillard, L.; Charra, F. Selective Excitation of Individual Plasmonic Hotspots at the Tips of Single Gold Nanostars. Nano Lett. 2011, 11 (2), 402-407.

(28) Awada, C.; Popescu, T.; Douillard, L.; Charra, F.; Perron, A.; Yockell-Lelièvre, H.; Baudrion, A.-L.; Adam, P.-M.; Bachelot, R. Selective Excitation of Plasmon Resonances of Single Au Triangles by Polarization-Dependent Light Excitation. J. Phys. Chem. C 2012, 116 (27), 14591-14598.

(29) Yurtsever, A.; Zewail, A. H. Direct Visualization of near-Fields in Nanoplasmonics and Nanophotonics. Nano Lett. 2012, 12 (6), 3334-3338.

(30) Schubert, O.; Becker, J.; Carbone, L.; Khalavka, Y.; Provalska, T.; Zins, I.; Sönnichsen, C. Mapping the Polarization Pattern of Plasmon Modes Reveals Nanoparticle Symmetry. Nano Lett. 2008, 8 (8), 2345-2350.

(31) Schön, P.; Bonod, N.; Devaux, E.; Wenger, J.; Rigneault, H.; Ebbesen, T. W.; Brasselet, S. Enhanced Second-Harmonic Generation from Individual Metallic Nanoapertures. Opt. Lett. 2010, 35 (23), 4063-4065.

(32) Brasselet, S. Polarization-Resolved Nonlinear Microscopy: Application to Structural Molecular and Biological Imaging. Adv. Opt. Photonics 2011, 3 (3), 205.

(33) Kauranen, M.; Zayats, A. V. Nonlinear Plasmonics. Nat. Photonics 2012, 6, 737-748.

(34) Shen, H.; Nguyen, N.; Gachet, D.; Maillard, V.; Toury, T.; Brasselet, S. Nanoscale Optical Properties of Metal Nanoparticles Probed by Second Harmonic Generation Microscopy. Opt. Express 2013, 21 (10), 12318-12326.

(35) Czaplicki, R.; Mäkitalo, J.; Siikanen, R.; Husu, H.; Lehtolahti, J.; Kuittinen, M.; Kauranen, M. SecondHarmonic Generation from Metal Nanoparticles: Resonance Enhancement versus Particle Geometry. Nano Lett. 2015, 15 (1), 530-534.

(36) Valev, V. K.; Silhanek, A. V; Smisdom, N.; De Clercq, B.; Gillijns, W.; Aktsipetrov, O. A.; Ameloot, M.; 
Moshchalkov, V. V; Verbiest, T. Linearly Polarized Second Harmonic Generation Microscopy Reveals Chirality. Opt. Express 2010, 18 (8), 8286-8293.

(37) Ghenuche, P.; Cherukulappurath, S.; Taminiau, T. H.; Van Hulst, N. F.; Quidant, R. Spectroscopic Mode Mapping of Resonant Plasmon Nanoantennas. Phys. Rev. Lett. 2008, 101 (11).

(38) Black, L. J.; Wiecha, P. R.; Wang, Y.; De Groot, C. H.; Paillard, V.; Girard, C.; Muskens, O. L.; Arbouet, A. Tailoring Second-Harmonic Generation in Single L-Shaped Plasmonic Nanoantennas from the Capacitive to Conductive Coupling Regime. ACS Photonics 2015, 2 (11), 1592-1601.

(39) Duboisset, J.; Aït-Belkacem, D.; Roche, M.; Rigneault, H.; Brasselet, S. Generic Model of the Molecular Orientational Distribution Probed by Polarization-Resolved Second-Harmonic Generation. Phys. Rev. A 2012, 85 (4), 43829.

(40) Richards, B.; Wolf, E. Electromagnetic Diffraction in Optical Systems. II. Structure of the Image Field in an Aplanatic System. Proc. R. Soc. London A Math. Phys. Eng. Sci. 1959, 253 (1274), 358379.

(41) Schön, P.; Behrndt, M.; Aït-Belkacem, D.; Rigneault, H.; Brasselet, S. Polarization and Phase Pulse Shaping Applied to Structural Contrast in Nonlinear Microscopy Imaging. Phys. Rev. A 2010, 81 (1), 13809.

(42) Réfrégier, P.; Roche, M.; Brasselet, S. Precision Analysis in Polarization-Resolved Second Harmonic Generation Microscopy. Opt. Lett. 2011, 36 (11), 2149-2151.

(43) Ferrand, P.; Gasecka, P.; Kress, A.; Wang, X.; Bioud, F.-Z.; Duboisset, J.; Brasselet, S. Ultimate Use of Two-Photon Fluorescence Microscopy to Map Orientational Behavior of Fluorophores. Biophys. J. 2014, 106 (11), 2330-2339.

(44) Balla, N. K.; So, P. T. C.; Sheppard, C. J. R. Second Harmonic Scattering from Small Particles Using Discrete Dipole Approximation. Opt. Express 2010, 18 (21), 21603-21611.

(45) Balla, N. K.; Yew, E. Y. S.; Sheppard, C. J. R.; So, P. T. C. Coupled and Uncoupled Dipole Models of Nonlinear Scattering. Opt. Express 2012, 20 (23), 25834-25842.

(46) Sandeau, N.; Le Xuan, L.; Chauvat, D.; Zhou, C.; Roch, J.-F.; Brasselet, S. Defocused Imaging of Second Harmonic Generation from a Single Nanocrystal. Opt. Express 2007, 15 (24), 1605116060.

(47) Hafi, N.; Grunwald, M.; van den Heuvel, L. S.; Aspelmeier, T.; Chen, J.-H.; Zagrebelsky, M.; Schütte, O. M.; Steinem, C.; Korte, M.; Munk, A.; Walla, P. J. Fluorescence Nanoscopy by Polarization Modulation and Polarization Angle Narrowing. Nat. Methods 2014, 11 (5), 579-584.

(48) Hentschel, M.; Saliba, M.; Vogelgesang, R.; Giessen, H.; Alivisatos, A. P.; Liu, N. Transition from Isolated to Collective Modes in Plasmonic Oligomers. Nano Lett. 2010, 10 (7), 2721-2726.

(49) Fan, J. A.; Bao, K.; Wu, C.; Bao, J.; Bardhan, R.; Halas, N. J.; Manoharan, V. N.; Shvets, G.; Nordlander, P.; Capasso, F. Fano-like Interference in Self-Assembled Plasmonic Quadrumer Clusters. Nano Lett. 2010, 10 (11), 4680-4685.

(50) Taubert, R.; Hentschel, M.; Kästel, J.; Giessen, H. Classical Analog of Electromagnetically Induced Absorption in Plasmonics. Nano Lett. 2012, 12 (3), 1367-1371.

(51) Rahmani, M.; Lei, D. Y.; Giannini, V.; Lukiyanchuk, B.; Ranjbar, M.; Liew, T. Y. F.; Hong, M.; Maier, S. A. Subgroup Decomposition of Plasmonic Resonances in Hybrid Oligomers: Modeling the Resonance Lineshape. Nano Lett. 2012, 12 (4), 2101-2106.

(52) Vercruysse, D.; Sonnefraud, Y.; Verellen, N.; Fuchs, F. B.; Di Martino, G.; Lagae, L.; Moshchalkov, V. V.; Maier, S. A.; Van Dorpe, P. Unidirectional Side Scattering of Light by a Single-Element Nanoantenna. Nano Lett. 2013, 13 (8), 3843-3849.

(53) Hancu, I. M.; Curto, A. G.; Castro-López, M.; Kuttge, M.; Van Hulst, N. F. Multipolar Interference for Directed Light Emission. Nano Lett. 2014, 14 (1), 166-171.

(54) Padmanabhan, P.; Thomas, K. G. Dimers of Au Nanorods. Adv Mater 2008, 20 (22), 4300-4305.

(55) Yang, Z.-J.; Zhang, Z.-S.; Zhang, L.-H.; Li, Q.-Q.; Hao, Z.-H.; Wang, Q.-Q. Fano Resonances in DipoleQuadrupole Plasmon Coupling Nanorod Dimers. Opt. Lett. 2011, 36 (9), 1542-1544.

(56) Kumar, J.; Wei, X.; Barrow, S.; Funston, A. M.; Thomas, K. G.; Mulvaney, P. Surface Plasmon 
Coupling in End-to-End Linked Gold Nanorod Dimers and Trimers. Phys. Chem. Chem. Phys. 2013, 15 (12), 4258-4264.

(57) Metzger, B.; Schumacher, T.; Hentschel, M.; Lippitz, M.; Giessen, H. Third Harmonic Mechanism in Complex Plasmonic Fano Structures. ACS Photonics 2014, 1 (6), 471-476.

(58) Ferrand, P. GPScan.VI: A General-Purpose LabVIEW Program for Scanning Imaging or Any Application Requiring Synchronous Analog Voltage Generation and Data Acquisition. Comput. Phys. Commun. 2015, 192, 342-347.

(59) Hao, F.; Nordlander, P. Efficient Dielectric Function for FDTD Simulation of the Optical Properties of Silver and Gold Nanoparticles. Chem. Phys. Lett. 2007, 446 (1-3), 115-118. 